\title{
Strain diversity and phage resistance in complex dairy starter cultures
}

\author{
M. Spus,${ }^{*} \dagger$ M. Li, ${ }^{\star} \dagger$ S. Alexeeva, ${ }^{*} \dagger$ J. C. M. Wolkers-Rooijackers,${ }^{*} \dagger$ M. H. Zwietering, $\dagger$ T. Abee, $\dagger$ \\ and E. J. Smid*t \\ ${ }^{*}$ TI Food and Nutrition, PO Box 557, 6700 AN Wageningen, the Netherlands \\ †Laboratory of Food Microbiology, Wageningen University, PO Box 17, 6700 AA Wageningen, the Netherlands
}

\begin{abstract}
The compositional stability of the complex Gouda cheese starter culture Ur is thought to be influenced by diversity in phage resistance of highly related strains that co-exist together with bacteriophages. To analyze the role of bacteriophages in maintaining culture diversity at the level of genetic lineages, simple blends of Lactococcus lactis strains were made and subsequently propagated for 152 generations in the absence and presence of selected bacteriophages. We first screened 102 single-colony isolates (strains) from the complex cheese starter for resistance to bacteriophages isolated from this starter. The collection of isolates represents all lactococcal genetic lineages present in the culture. Large differences were found in bacteriophage resistance among strains belonging to the same genetic lineage and among strains from different lineages. The blends of strains were designed such that 3 genetic lineages were represented by strains with different levels of phage resistance. The relative abundance of the lineages in blends with phages was not stable throughout propagation, leading to continuous changes in composition up to 152 generations. The individual resistance of strains to phage predation was confirmed as one of the factors influencing starter culture diversity. Furthermore, loss of proteolytic activity of initially proteolytic strains was found. Reconstituted blends with only 4 strains with a variable degree of phage resistance showed complex behavior during prolonged propagation.
\end{abstract}

Key words: starter culture, bacteriophage, diversity, proteolytic activity

\section{INTRODUCTION}

In cheese production, 2 types of starter cultures are used: undefined and defined. Undefined starters originate from successful artisanal cheese production processes and were traditionally propagated in milk by

Received March 5, 2015

Accepted April 11, 2015.

${ }^{1}$ Corresponding author: eddy.smid@wur.nl back-slopping (Stadhouders and Leenders, 1984; Wouters et al., 2002); with back-slopping, the milk is inoculated with a small portion of a previously performed successful fermentation. Generally, the composition of undefined starters is unknown, which leads to uncertainty in terms of their performance in milk (Daly et al., 1996). Defined starters are manufactured blends of two or more strains, which ensures consistency in product quality. Nevertheless, defined starter cultures are more susceptible to bacteriophage predation when used in cheese manufacturing, and bacteriophages are still a major cause of fermentation failures in the dairy industry, leading to substantial raw material losses (McGrath et al., 2007). The limited number of strains used in such cultures explains the higher bacteriophage sensitivity of defined blends compared with more complex undefined starters. On the other hand, complex starter cultures containing highly related strains with variable levels of phage sensitivity are expected to be more resistant to phage attack because the phage-sensitive strains in these cultures will be replaced by their phage-resistant counterparts upon phage attack (Erkus et al., 2013).

Previously, an undefined complex Gouda type cheese starter culture called Ur was characterized in detail (Erkus et al., 2013). This starter culture consists of only 2 lactic acid bacteria: Lactococcus lactis and Leuconostoc mesenteroides, encompassing in total 8 genetic lineages as determined by a high-resolution amplified fragment length polymorphism (AFLP)-based method (Kütahya et al., 2011). Complex culture isolates (strains) belonging to the same genetic lineage possess similar functionally relevant phenotypic characteristics. For instance, all strains belonging to lineages 1, 3, and 5 (all Lactococcus lactis ssp. cremoris) possess proteolytic activity, and all lactococcal strains with the ability to utilize citrate belong to lineages 2 and 4 (both Lactococcus lactis ssp. lactis biovar diacetylactis). Isolates from the same genetic lineage are expected to show similar behavior during cheese production. Based on the analysis of only a limited number of strains, Erkus and co-workers (2013) showed that different strains belonging to the same genetic lineage display a large variation in bacteriophage resistance when challenged 
with phages originating from the same complex starter culture. Moreover, individual strains isolated from the starter culture were found to differ in plasmid content (Erkus et al., 2013).

The compositional stability of the Ur starter culture is thought to be partially based on the diversity in phage resistance of highly related strains that co-exist with bacteriophages (Erkus et al., 2013). It has been suggested that bacteriophage predation can prevent domination of a microbial community by a single variant. This mechanism provides the basis of the constantdiversity (CD) dynamics model, described by Rodriguez-Valera et al. (2009). The CD model explains the maintenance of microbial community diversity through bacteriophage predation based on a "kill-the-winner" mechanism (Thingstad, 2000; Winter et al., 2010). This mechanism prevents domination of the community by the fittest variant, because abundance of this variant will be eventually reduced by the bacteriophage.

We hypothesize that when the microbial community of a starter culture, stratified in genetic lineages, possesses diversity at the strain level, the diversity at the level of genetic lineages will be maintained by bacteriophage predation. To investigate if this phenomenon also occurs in simple blends of strains, such reconstituted cultures were sequentially propagated in the absence and presence of selected bacteriophages. In our study, diversity is a function of the number of genetic lineages present in the blend and their relative abundance (Haegeman et al., 2013). Compared with the nonchallenged control blends, relative abundance of different genetic lineages was not stable in blends challenged with bacteriophages, demonstrating the effect of phages on diversity in simple blends.

\section{MATERIALS AND METHODS}

\section{Complex Starter Culture Isolates}

Single-colony isolates of the complex starter culture Ur used in this study were collected from LM17 agar plates (Oxoid, Basingstoke UK; $1.5 \%$ wt/vol) supplemented with lactose (Oxoid; $0.5 \%$ wt/vol; Terzaghi and Sandine, 1975) and Reddy agar plates as described previously by Erkus et al. (2013). Individual isolates were characterized and classified into 8 genetic lineages by AFLP typing (Kütahya et al., 2011): Lactococcus lactis ssp. cremoris (lineages 1, 3, 5, 6, and 7), Lactococcus lactis ssp. lactis biovar diacetylactis (lineages 2 and 4), and Leuconostoc mesenteroides ssp. cremoris (lineage 8 ). Similarity of the genetic profiles of $<90 \%$ was considered as the cut-off for defining separate genetic lineages. Isolates were maintained as glycerol stocks at $-80^{\circ} \mathrm{C}$ and reactivated, in the case of lactococci, in
LM17 broth (Oxoid) with addition of $0.5 \%$ (wt/vol) lactose (Oxoid) and, for Leuconostoc strains, in de Man, Rogosa, and Sharpe broth (Merck, Schiphol-Rij, the Netherlands).

\section{Determination of Growth Rate of Representative Complex Starter Isolates}

Complex culture isolates L. lactis TIFN1 (lineage 1), L. lactis TIFN2 (lineage 2), L. lactis TIFN3 (lineage 3), L. lactis TIFN4 (lineage 4), L. lactis TIFN5 (lineage 5), L. lactis TIFN6 (lineage 6), L. lactis TIFN7 (lineage 7), and Leu. mesenteroides TIFN8 (lineage 8) were used to determine specific growth rates in skim milk supplemented with $1 \%$ (wt/vol) casiton (Oxoid). Overnight cultures were washed by centrifugation at $5,000 \times g$ for $10 \mathrm{~min}$. The supernatant was discarded and pellets were resuspended in the same volume of magnesium sulfate $(5 \mathrm{mM})$-potassium phosphate (15 $\mathrm{m} M)$ buffer $(\mathrm{pH}=6.5)$. Washed cells were inoculated $(1 \% \mathrm{vol} / \mathrm{vol})$ into skim milk (Friesche Vlag Lang Lekker, non-fat, UHT, Friesland Campina, Amersfoort, the Netherlands) for growth experiments. Optical density at $600 \mathrm{~nm}\left(\mathbf{O D}_{600}\right)$ was measured hourly in a $1.5-\mathrm{mL}$ semi-micro cuvette (light path length $=1 \mathrm{~cm}$ ) using a spectrophotometer (Novaspec Plus, Biochrom, Cambridge, UK). Before measurement, milk samples were cleared with $0.2 \%$ (wt/vol) $\mathrm{NaOH}$ and $0.2 \%$ (wt/vol) Titriplex III (Merck) solution. Cleared skim milk was used as a reference. The specific growth rate was then calculated by plotting semi-logarithmically the $\mathrm{OD}_{600}$ values of exponential growth phase (logarithmic scale) versus time (linear scale); specific growth rates were expressed as $\mathrm{h}^{-1}$. Because of the clarification step, plate counting was used to verify the accuracy of the growth curve based on $\mathrm{OD}_{600}$ data. One milliliter of milk sample was added to $9 \mathrm{~mL}$ of peptone physiological salt solution containing $0.85 \%$ (wt/vol) $\mathrm{NaCl}$ and $0.1 \%$ (wt/vol) neutralized bacteriological peptone (Oxoid) to make the first dilution. Serial dilutions were made and $50 \mu \mathrm{L}$ of diluted sample was spread onto an LM17 agar plate or, for strain TIFN8, an MRSV agar plate (MRS supplemented with $30 \mathrm{mg} / \mathrm{mL}$ of vancomycin; Duchefa Biochemie, Haarlem, the Netherlands) using a spiral plater. Plates were then incubated at $30^{\circ} \mathrm{C}$ (LM17) or $25^{\circ} \mathrm{C}$ (MRSV) for $48 \mathrm{~h}$ and colonies were counted.

\section{Sequential Propagation of Simple Defined Blends}

Blends used as an inoculum for sequential propagation were prepared from overnight cultures of selected isolates (for details, see Figure 1B). Optical density of the overnight isolates cultures was measured and 
cultures were diluted further to obtain the same cell density: $\mathrm{OD}_{600}=1.1$. Bacterial cells were centrifuged $(10,000 \times g, 3 \mathrm{~min})$ and resuspended in magnesium sulfate $(5 \mathrm{~m} M)$-potassium phosphate $(15 \mathrm{mM})$ buffer $(\mathrm{pH}$ $6.5)$. Next, cells were centrifuged again and resuspended in 10 times less buffer. Defined blends of strains were inoculated (1\%) in $10 \mathrm{~mL}$ of skim milk (Friesche Vlag Lang Lekker, non-fat, UHT milk, Friesland Campina). Each 24 h, 0.5\% (vol/vol) of the previous day's milk culture was used as an inoculum for propagation in fresh milk, obtaining 7.6 generations after each transfer. Blends were incubated at $20^{\circ} \mathrm{C}$. Samples of the blends were taken each $48 \mathrm{~h}$ and kept frozen at $-20^{\circ} \mathrm{C}$ until further analysis. Where relevant, bacteriophages were added to the blends at a concentration of $10^{8} \mathrm{pfu} / \mathrm{mL}$.

\section{Bacteriophages}

Bacteriophages used in this study were isolated as described in Erkus et al. (2013). Three phages found in the supernatant of the undefined starter culture were labeled as $\Phi$ TIFN1, $\Phi T I F N 5$, and $\Phi T I F N 7$. Based on genome sequence information, $\Phi$ TIFN1 and $\Phi$ TIFN7 belong to the lactococcal phage type P335, whereas TIFN5 is classified as a type 936 lactococcal phage (Mahony and van Sinderen, 2014).

\section{Viable Plate Counting}

Propagated blends of L. lactis strains were plated on $\beta$-glycerolphosphate-buffered milk agar (GMA) plates supplemented with bromocresol purple (Huggins and Sandine, 1984). Samples of cultured blends were diluted and plated in duplicate. The differential plating was used to estimate the loss of protease activity in the sequentially propagated blends. Protease-positive colonies are larger and are surrounded by a yellow zone compared with the small, translucent colonies of protease-negative variants.

\section{Quantitative PCR}

As described by Erkus et al. (2013), representative strains TIFN1, TIFN2, TIFN3, TIFN4, TIFN5, TIFN6, TIFN7, and TIFN 8 of the 8 genetic lineages were selected and their genomes sequenced. Primer sets were developed based on sequences of unique genes of each representative strain (Supplemental Table S1; http:// dx.doi.org/10.3168/jds.2015-9535) to obtain amplicon lengths of 60 to $70 \mathrm{bp}$. Primers were optimized for consistent annealing at $60^{\circ} \mathrm{C}$ for similar reaction conditions. Pairwise comparison of the genomes of strains TIFN1 and TIFN5 and of strains TIFN2 and TIFN4 showed only a limited number of strain-specific unique genes. None of those unique genes met all the criteria set for designing specific primers. To enumerate lineage 1 and 5 strains, probes were designed that reacted specifically with strain TIFN1 as well as strain TIFN5 (hereafter, lineage 1-5). To enumerate lineage 2 and 4 strains, probes were designed that reacted specifically with strain TIFN2 as well as strain TIFN4. Samples of the propagated blends were collected after $24 \mathrm{~h}$ and subsequently each $48 \mathrm{~h}$ (every 2 transfers), and lineage-specific quantitative (q)PCR was performed to determine the relative abundance of copy numbers of each lineage in the blend.

\section{Colony PCR}

Primers for colony PCR (Supplemental Table S2; http://dx.doi.org/10.3168/jds.2015-9535) were designed based on the same collection of unique genes as described in the $\mathrm{qPCR}$ approach. Primers were designed so that the obtained PCR products would differ by approximately $80 \mathrm{bp}$ to allow distinction by gel electrophoresis. A colony was picked from the GMA plate using a sterile loop and directly transferred to the PCR mix. PCR mix contained $5 \mu \mathrm{L}$ of dNTP $(2 \mathrm{~m} M), 2 \mu \mathrm{L}$ of forward primer $(10 \mu M), 2 \mu \mathrm{L}$ of reverse primer $(10$ $\mu M), 5 \mu \mathrm{L}$ of $10 \times$ DreamTaq buffer (Thermo Fisher Scientific, Waltham, MA), $3 \mu \mathrm{L}$ of $\mathrm{MgCl}_{2}(25 \mathrm{mM})$, and $0.4 \mu \mathrm{L}$ of DreamTaq DNA Polymerase (Thermo Fisher Scientific; $5 \mathrm{U} / \mu \mathrm{L})$. The program of the PCR run contained the following steps: $10 \mathrm{~min}$ at $95^{\circ} \mathrm{C}$ for cell lysis and activation of DNA polymerase, 25 cycles of $30 \mathrm{~s}$ at $95^{\circ} \mathrm{C}$ for DNA melting, $20 \mathrm{~s}$ at $60^{\circ} \mathrm{C}$ for primer annealing, and $30 \mathrm{~s}$ at $72^{\circ} \mathrm{C}$ for elongation. The PCR reaction finished with $4 \mathrm{~min}$ at $72^{\circ} \mathrm{C}$ for final elongation.

\section{Phage Resistance by Spot Assay}

Phage resistance of 102 single-colony isolates of the undefined complex starter culture Ur was determined using a qualitative spot assay (Sanders and Klaenhammer, 1983). Modifications of the method included smaller Petri dish plates containing LM17 agar $[35 \mathrm{~mm}$ in diameter, $1.5 \%$ (wt/vol) agar, supplemented with $0.5 \%$ (wt/vol) lactose and $10 \mathrm{mM} \mathrm{CaCl}$; Greiner BioOne, Alphen aan den Rijn, the Netherlands], a lesser volume of soft layer agar $(1 \mathrm{~mL})$, and a lesser volume of highly concentrated $\left(10^{10} \mathrm{pfu} / \mathrm{mL}\right)$ phage solution $(5 \mu \mathrm{L})$.

\section{Plaque Assay}

The plaque assay used in this study was a slight modification of the method described by Lillehaug (1997). 
A

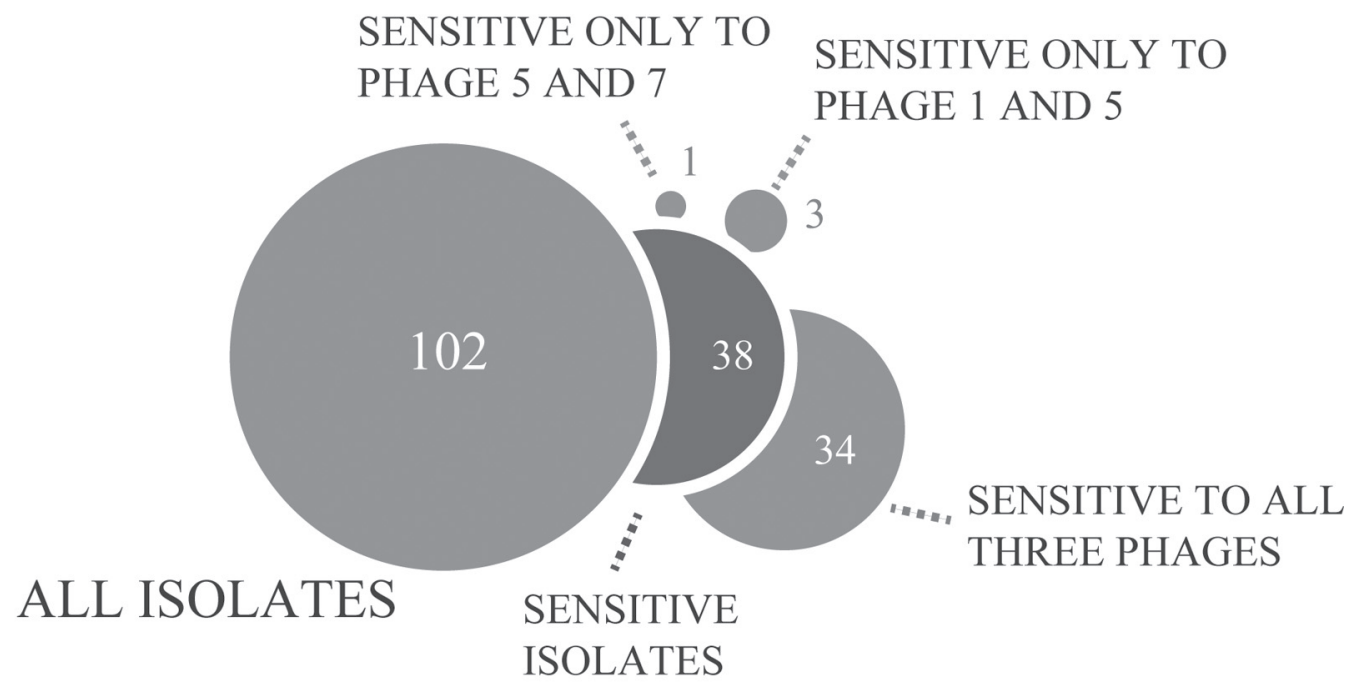

B

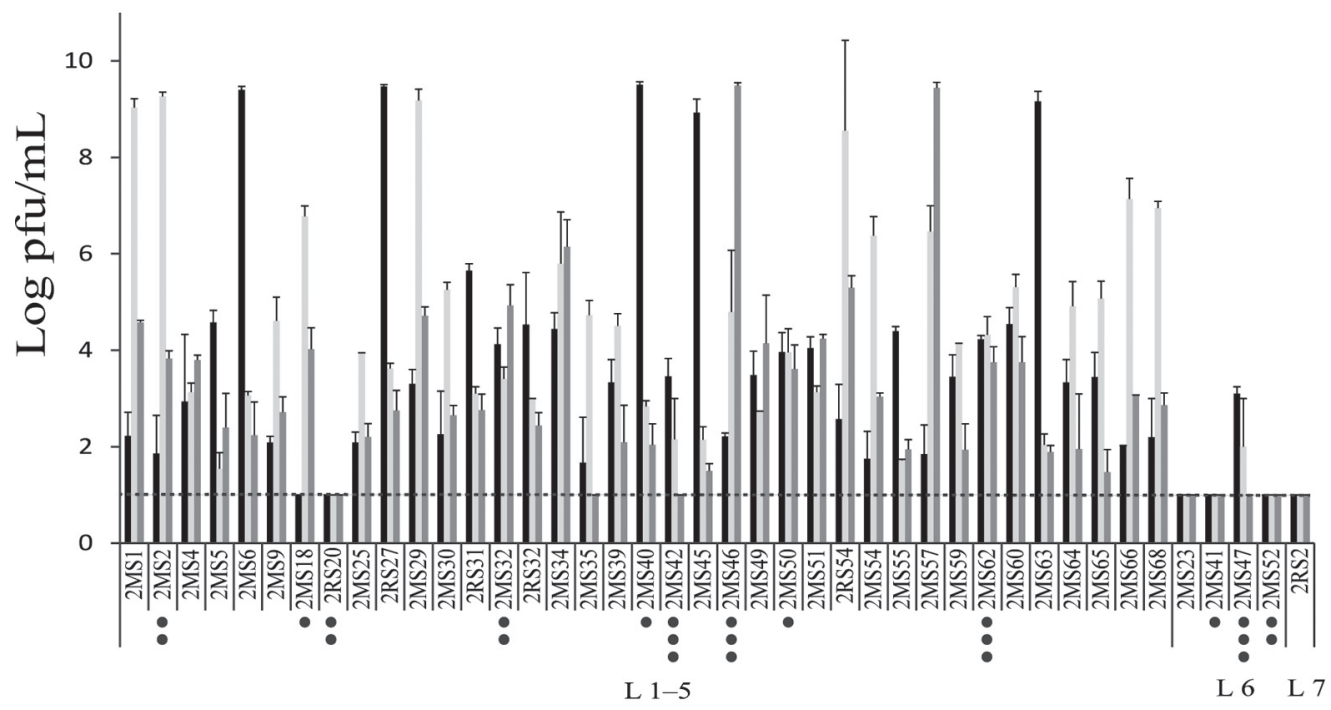

Starter isolates

Figure 1. (A) Summary of bacteriophage resistance survey of $102 \mathrm{Ur}$ starter isolates based on spot assay results. (B) Phage titer determination of single-colony isolates of Ur starter culture; error bars represent standard deviations. All strains that were sensitive in the spot assay were tested plus 4 strains that showed complete resistance in the spot assay (in total 42 strains). L $1-5=$ lineage $1-5 ; \mathrm{L} 6=$ lineage $6 ; \mathrm{L} 7=$ lineage 7. Strains used in blend $1=1$ black dot; strains used in blend $2=2$ black dots; strains used in blend $3=3$ black dots. Dashed line represents detection limit for phage titer determination method $(1 \log \mathrm{pfu} / \mathrm{mL})$. Black bars $=$ results for phage $\Phi$ TIFN1; light gray bars $=$ results for phage $\Phi$ TIFN5; = dark gray bars $=$ results for phage $\Phi$ TIFN7.

One hundred microliters of phage solution diluted in a phage buffer [Tris- $\mathrm{HCl} \mathrm{pH} 7.5$ (final concentration 50 $\mathrm{m} M$ ), $\mathrm{NaCl}$ (final concentration $100 \mathrm{mM}$ ), $\mathrm{CaCl}_{2}$ (final concentration $5 \mathrm{mM}$ ), $\mathrm{MgSO}_{4}$ (final concentration $1 \mathrm{mM}$ ), gelatin (final concentration $0.01 \%(\mathrm{wt} / \mathrm{vol})]$ and sterile water was added to $100 \mu \mathrm{L}$ of the overnight culture of a single-colony isolate of a query strain. The mixture was kept for $10 \mathrm{~min}$ at $37^{\circ} \mathrm{C}$ in a water bath, mixed with $3 \mathrm{~mL}$ of top soft agar $(0.75 \% \mathrm{wt} / \mathrm{vol})$, and finally poured on LM17 agar plates. After 24-h incubation at $30^{\circ} \mathrm{C}$, plaques were counted and plaque-forming units per milliliter values were calculated. 


\section{RESULTS}

\section{Growth Rates of Individual Strains of Complex Starter}

Individual strains isolated from the complex starter culture and representing all genetic lineages present in the culture were found to differ in their maximum growth rates $\left(\boldsymbol{\mu}_{\max }\right)$ in casiton-fortified milk. Strain TIFN5 (representative of lineage 5) had the highest growth rate $\left(\mu_{\max }=0.67 \mathrm{~h}^{-1}\right)$, followed by strain TIFN1 (lineage $1 ; \mu_{\max }=0.58 \mathrm{~h}^{-1}$ ), strain TIFN8 (lineage $8 ; \mu_{\max }=0.5 \mathrm{~h}^{-1}$ ), strain TIFN3 (lineage $3 ; \mu_{\max }$ $=0.49 \mathrm{~h}^{-1}$ ), strain TIFN4 (lineage $4 ; \mu_{\max }=0.41 \mathrm{~h}^{-1}$ ), strain TIFN7 (lineage $7 ; \mu_{\max }=0.31 \mathrm{~h}^{-1}$ ), strain TIFN2 (lineage $2 ; \mu_{\max }=0.29 \mathrm{~h}^{-1}$ ) and, finally, strain TIFN6 (lineage $6 ; \mu_{\max }=0.24 \mathrm{~h}^{-1}$ ). Based solely on these measured growth rate differences, the slow-growing lineage 6 , for instance, is expected to disappear from the culture after 5 transfers (46 generations; see Supplemental Figure S1; http://dx.doi.org/10.3168/jds.2015-9535). The co-existence of different strains in the starter despite differences in individual growth rates suggests the influence of other factors in maintaining strain diversity, such as microbe-microbe interactions (Smid and Lacroix, 2013), selective conditions in acidifying milk, or phage predation.

\section{In-Depth Analysis of Phage Resistance Profiles of 102 Individual Isolates}

To obtain a detailed overview of the level of bacteriophage resistance of members of the complex starter culture, we determined the bacteriophage resistance of 102 strains (single-colony isolates), representing the 7 lactococcal genetic lineages of the starter culture, was determined using a spot test assay using the 3 phages ( $\Phi$ TIFN1, $\Phi$ TIFN5, and $\Phi$ TIFN7) previously isolated from Ur (Erkus et al., 2013; Supplemental Figure S2; http://dx.doi.org/10.3168/jds.2015-9535). This screening demonstrated that 38 out of 102 strains tested were sensitive to at least 1 of the 3 bacteriophages used in this study (Figure 1A). These 38 sensitive strains together with 4 resistant strains (in total 42 ) were subsequently used for quantitative phage titer determination. A large variation was found in bacteriophage resistance among the 42 isolates (Figure 1B). First, differences in resistance for one particular phage were found between isolates belonging to different genetic lineages. Interestingly, very large differences in resistance were detected between isolates belonging to the same genetic lineage, ranging from values below the detection limit $(10 \mathrm{pfu} / \mathrm{mL})$ to $10^{9} \mathrm{pfu} / \mathrm{mL}$. Large differences in phage resistance of closely related $L$. lactis strains have been reported previously (Ward et al., 2004). Based on results of the quantitative phage resistance assay, the collection of strains was classified into 4 resistance groups: (1) sensitive (S), >6 log pfu/mL; (2) moderately resistant (MR), between 3 and $6 \log \mathrm{pfu} / \mathrm{mL} ;(3)$ resistant $(\mathbf{R})$, between 1 and $3 \log \mathrm{pfu} / \mathrm{mL}$; and (4) no detectable susceptibility (ND), $<1$ log pfu/mL (detection limit). Results of the phage resistance analysis can be used to design blends of strains with a known degree of phage resistance. This also allows us to determine experimentally if relatively simple blends (composed of 4 strains representing 3 lineages) meet the required level of culture diversity for stabilizing the community diversity driven by a CD-like mechanism.

\section{Design of Defined Blends of Strains with Variation in Phage Resistance}

The next step was to determine the effect of bacteriophage predation on the maintenance of diversity of simple blends of starter isolates upon sequential propagation. For that reason, we designed simple blends of strains from lineage $1-5$ and lineage 6 with a known degree of phage resistance (Supplemental Table S3; http://dx.doi.org/10.3168/jds.2015-9535). In the experimental design (Figure 2), we (1) reduced the complexity of the starter culture by limiting the number of strains used in blends, and (2) mimicked the variation in resistance to phages found in the starter culture. These 2 requirements were met by using only 4 strains inoculated in equal amounts: 3 representatives from lineage $1-5$ and 1 representative from lineage 6 . Three strains from lineage 1-5 differ in their degree of resistance to phages: S, MR, and R. No detectable sensitivity (ND) was found for the strain belonging to lineage 6 . The strains from lineage $1-5$ possessed caseinolytic activity (protease positive, prt $^{+}$) and the strains from lineage 6 were protease negative $\left(\mathbf{p r t}^{-}\right)$. In total, 3 blends (blends 1, 2, and 3) were prepared that were challenged at the onset of the experiment with phage $\Phi T I F N 1, \Phi T I F N 5$, or $\Phi T I F N 7$, respectively (Supplemental Table S3). Relative abundance at the level of genetic lineages was monitored throughout the propagation experiment using a qPCR-based method (see Materials and Methods). Control blends were not challenged with phages.

\section{Population Dynamics in Defined Blends}

All blends described in the previous paragraph were propagated in milk for 152 generations and the relative abundance of lineage 1-5 and lineage 6 was determined during the course of the experiment (Figure 3). 


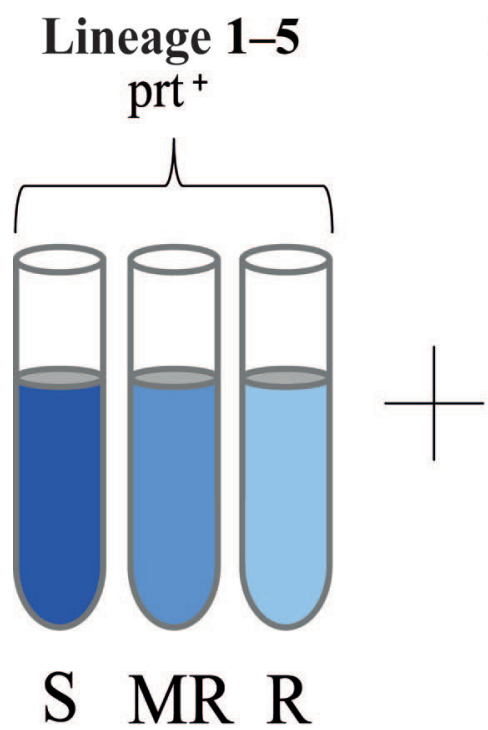

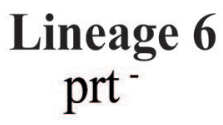

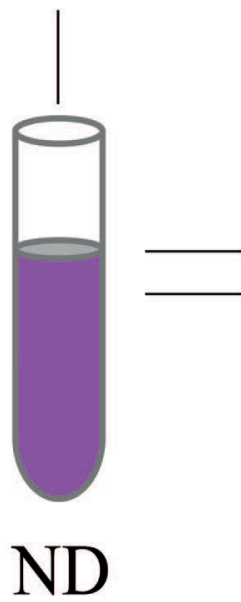

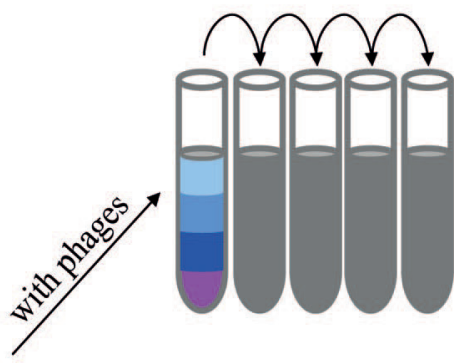

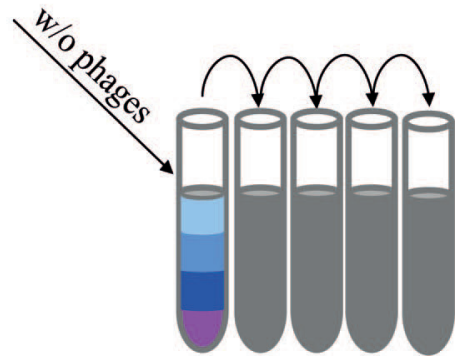

Figure 2. Experimental design of simple blends. $\mathrm{S}=$ sensitive isolate $(>6 \log \mathrm{pfu} / \mathrm{mL}) ; \mathrm{MR}=$ moderately resistant isolate $(>3,<6 \log$ pfu/

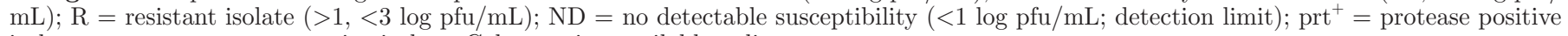
isolates; $\mathrm{prt}^{-}=$protease negative isolate. Color version available online.

In all designed blends, the initial distribution of lineages (75\% lineage $1-5 ; 25 \%$ lineage 6$)$ changed after 1 propagation cycle and, in all cases, one of the lineages became dominant. In blends without phage addition, lineage 1-5 became dominant, with relative abundances ranging from $79 \%$ (blend 3) to $99 \%$ (blend 1). In the phage-challenged blend 2 and blend 3 , lineage 6 developed dominance with more than $99 \%$ relative abundance in the culture. One exception was the phage-challenged blend 1 , in which lineage $1-5$ was detected at a relative abundance of $97 \%$. In nonchallenged blends, lineage 1-5 retained dominance throughout the entire propagation experiment, with only one deviating observation - in blend 2 after 91 generations. Phage-challenged blends were overall more dynamic in composition compared with the nonchallenged blends. In blend 1, lineage 1-5 decreased and then increased, whereas in blends 2 and 3, initial abundance of lineage 6 was reversed after about 40 generations.

\section{$\mathrm{Prt}^{+} / \mathrm{Prt}^{-}$Shifts Can Be Explained by Competition and Loss of Plasmid Encoding prtP Gene}

The initial percentage of protease-positive strains in all 3 blends was set at $75 \%$ (contributed by 3 lineage 1-5 strains). At the end of the propagation experiment, we could still detect protease-positive colony forming units but the initial percentage was reduced substantially. For blend 1, challenged with phage $\Phi T I F N 1$, prt $^{+}$colonies averaged $8 \%$ (while $60 \%$ lineage $1-5$ ) and, without phage challenge, averaged 69\% (99\% lineage $1-5)$. For blend 2, challenged with phage $\Phi$ TIFN5, prt $^{+}$ colonies averaged $65 \%$ (77\% lineage 1-5) and without challenge, averaged $51 \%$ (90\% lineage 1-5). For blend 3, challenged with phage $\Phi$ TIFN7, prt $^{+}$colonies averaged $27 \%$ (95\% lineage 1-5) and, without phage challenge, averaged 32\% (98\% lineage 1-5; Supplemental Table S4; http://dx.doi.org/10.3168/2015-9535).

After 152 generations (end-point of sequential propagation), we isolated $10 \mathrm{prt}^{+}$colonies and $10 \mathrm{prt}^{-}$colonies from blends challenged with bacteriophages and performed colony PCR with lineage-specific primer sets. Interestingly, in blends 2 and 3 , all prt ${ }^{-}$colonies were found to belong to lineage 1-5 (data not shown). It is known that upon propagation in milk, prt $^{+}$variants can lose the plasmid that carries the gene encoding the caseinolytic protease (prtP; McKay and Baldwin, 1975; Laan et al., 1989). The loss of plasmids can give a growth advantage to the host (higher growth rate) in competition for niche domination (Hugenholtz and Veldkamp, 1985; Bachmann et al., 2011).

\section{Phage Sensitivity After Sequential Propagation}

We exposed the 60 single-colony isolates described previously (originating from the end-point of sequential propagation; 20 per blend) to the filtered supernatant collected from the blends at the same time point as well 
I

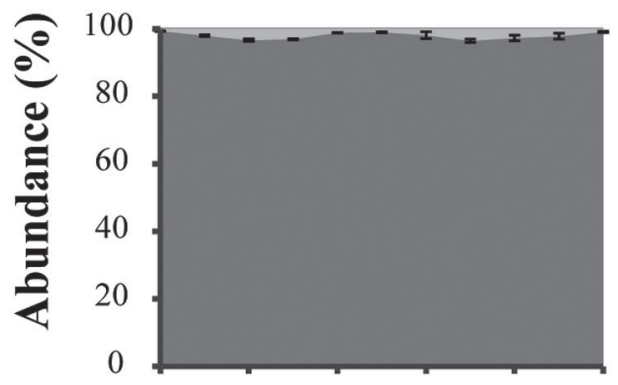

III

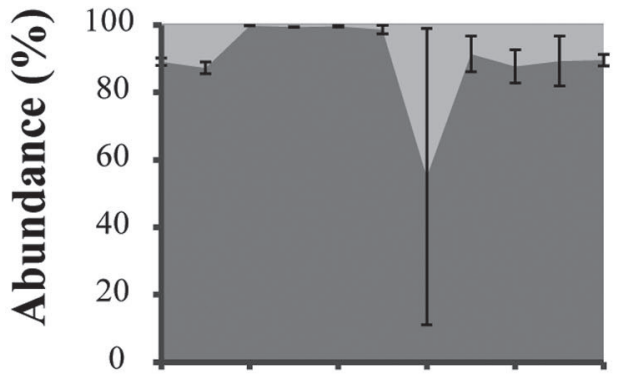

V

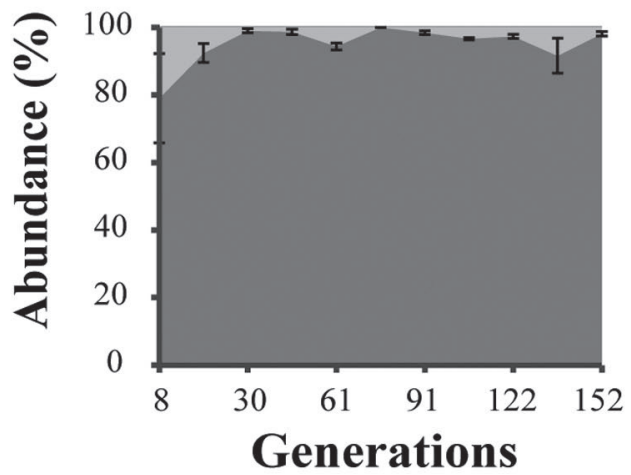

- Lineage 1-5
II

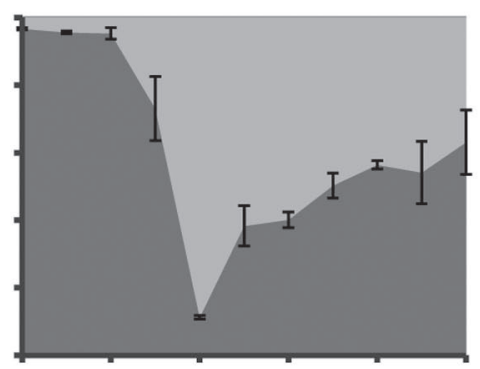

IV

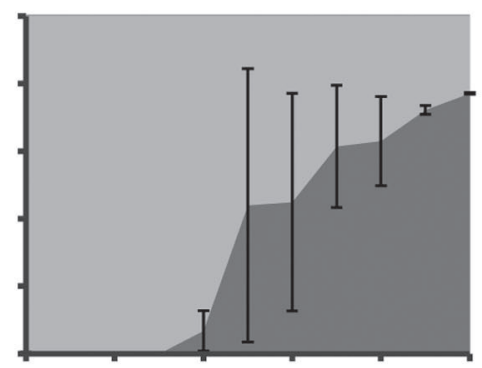

VI

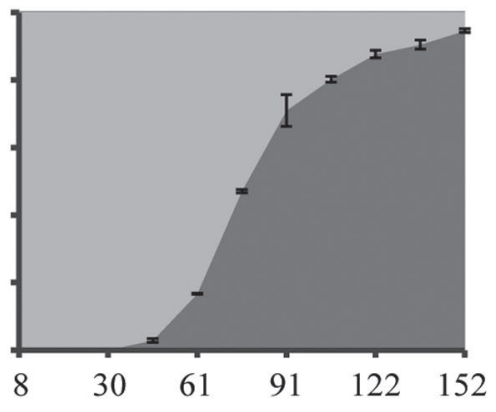

Generations

Lineage 6

Figure 3. Relative abundance of lineages in blends during propagation experiment. Dark gray $=$ lineage $1-5$; light gray $=$ lineage 6 ; $\mathrm{I}=$ blend 1 without phage; II = blend 1 with $\Phi$ TIFN1; III = blend 2 without phage; IV = blend 2 with $\Phi$ TIFN5; V = blend 3 without phage; VI = blend 3 with $\Phi$ TIFN7. Black bars represent standard deviation of 2 independent biological replicates; error bars represent standard deviations.

as to the original bacteriophages $\Phi$ TIFN1, $\Phi$ TIFN5 and $\Phi$ TIFN7 (Supplemental Figure S3; http://dx.doi. org/10.3168/jds.2015-9535). Only 2 isolates were sensitive to the phages present in the supernatant; these came from blend 1 . These 2 isolates were also sensitive to $\Phi$ TIFN1. Although none of the single-colony isolates from blends 2 and 3 showed plaques when exposed to the corresponding end-point supernatants, they were all sensitive towards the original bacteriophages ( $\Phi$ TIFN5 and $\Phi$ TIFN7, respectively).

\section{DISCUSSION}

In this study, we demonstrated in detail the large variation in the degree of phage resistance among strains isolated from a complex cheese starter culture representing different lineages and, more importantly, among strains originating from the same lineage. This variation is thought to be instrumental for phagemediated compositional stability of microbial communities because it theoretically prevents clonal sweeps 
of genetic lineages. Similar observations were reported in a study of Cellulophaga baltica in the marine environments by Holmfeldt et al. (2007), who showed that large differences in phage resistance are present among highly related strains of $C$. baltica, indicating the highly complex character of phage-host interactions in this environment. Holmfeldt et al. (2007) suggested that high variation in resistance to phages assures continuous succession of less-susceptible strains and supports the hypothesized role of phages as drivers of strain diversity and their impact on population dynamics.

In this study, the genetic lineage diversity of a complex starter culture was investigated by designing blends of well-characterized culture isolates. This approach enabled us to assess the effect of bacteriophages on diversity at the level of genetic lineages in simple blends of well-characterized L. lactis strains.

In blends without phage addition (i.e., control blends), lineage 1-5 (phage sensitive and protease positive) dominated the community, most likely due to higher growth rate in milk compared with that of the strain representing lineage 6 . This observation is in accordance with our predictions based on the specific growth rates of the representative strains (Supplemental Figure S1; http://dx.doi.org/10.3168/jds.2015-9535). After 1 propagation cycle of conjoint growth of strain TIFN1 with strain TIFN6, TIFN1 would reach $9 \mathrm{log}$ (94\% total abundance) and TIFN6 would reach $7.8 \mathrm{log}$ (6\% total abundance). Based on the observed growth rates, when strains TIFN5 and TIFN6 are co-cultured, they would reach $9 \log$ (96\% total abundance) and 7.7 $\log$ (4\% total abundance), respectively. These theoretical calculations are comparable to the experimental results, where after 1 propagation cycle, the measured abundance of lineage 1-5 ranged from $79 \%$ (blend 3) to $99 \%$ (blend 1). It has been reported previously that $\mathrm{prt}^{+}$strains of L. lactis have higher growth rates compared with $\mathrm{prt}^{-}$strains at $\mathrm{pH}$ values $<6$ (Hugenholtz and Veldkamp, 1985), a $\mathrm{pH}$ value typical of acidifying milk. Moreover, the phenomenon of high local substrate availability stabilizing cooperative traits such as the expression of an extracellular protease (Bachmann et al., 2011) conceivably contributes to the dominance of lineage 1-5 in blends without phage. In this model, the outcome of the competition between $\mathrm{prt}^{+}$and $\mathrm{prt}^{-}$is density dependent. Protease-negative strains can only outcompete $\mathrm{prt}^{+}$strains at high cell densities when high global concentrations of peptides prevail. After 152 generations of sequential propagation (final time point in our experiment), we found prt $^{-}$variants belonging to lineage 1-5. Apparently, a fraction of the $\mathrm{prt}^{+}$cells of lineage 1-5 lost the plasmid carrying the protease-encoding gene (McKay and Baldwin, 1975). The newly emerging prt $^{-}$variants substituted part of nonproteolytic lineage 6 variants in the control blends, leading to stable cooperation between the prt $^{+}$fraction of lineage $1-5$, the prt $^{-}$fraction of lineage $1-5$, and the prt $^{-}$lineage 6 strains. In blend 1 without phage, $99 \%$ of the abundance was ascribed to strains of lineage $1-5$ but we found only $69 \%$ of the colonies to be $\mathrm{prt}^{+}$. For blend 2, strains of lineage 1-5 comprised $90 \%$ of total abundance but only $51 \%$ of all colonies were prt $^{+}$, and for blend 3 , lineage 1-5 dominated with $98 \%$ abundance but we found only $32 \% \mathrm{prt}^{+}$colonies.

In the phage-challenged blends, we observed 2 trends in the population dynamics: (1) initial dominance of lineage 1-5 followed by a decrease in its abundance and finally a steady increase (Figure 3, panel II) and (2) initial dominance of lineage 6 through the first 46 generations followed by a steady increase in abundance of lineage 1-5 (Figure 3, panels IV and VI). Both phenomena are probably influenced by phage predation because we did not observe them in the control blends. In the phage-challenged blend 1, most likely the phage-resistant strain (R) of lineage $1-5$ dominated the blend until 30 generations, followed by a decrease until 61 generations. The latter might be explained by the occurrence of (evolved) variants of phage $\Phi T I F N 1$ with increased lytic activity towards strain 2MS18 (the $\mathrm{R}$ variant of lineage $1-5)$. It is known that phages can adapt quickly to new potential hosts (Gómez and Buckling, 2011). Because many prophage sequences have been found in the genomes of sequenced Ur isolates (Erkus et al., 2013), an alternative explanation might be the expression of prophages during the propagation experiment, potentially releasing phages that predate on the resistant variants in the blend. Moreover, individual strains isolated from Ur and belonging to genetic lineage 1-5 were found to contain inducible prophages (our unpublished results). Finally, the subsequent increase in abundance of lineage 1-5 variants might reflect an increase in numbers of $\mathrm{MR}$ or $\mathrm{S}$ strains due to inability of the expressed prophage to predate on these strains.

When we challenged blends 2 and 3 with $\Phi$ TIFN5 and TTIFN7, respectively (Figure 3, panels IV and VI), initially only a small fraction of lineage $1-5$ variants $(<1 \%)$ survived predation by these phages. Both blends were initially dominated by the $\mathrm{prt}^{-}$lineage 6 strain, feeding on the peptides and amino acids released by the large number of lysed cells or by the minor fraction of proteolytic survivors belonging to lineage 1-5. The subsequent increase in abundance of lineage $1-5$ strain(s) observed after 46 generations can be ascribed to the occurrence of variants with increased phage resistance or to "wash out" of phages after disappearance of phage-sensitive host cells in the culture. In blends 2 and 3 , lineage $1-5$ variants gradually increased in 
abundance after 46 generations (i.e., 5 transfers), with each transfer ( $0.5 \%$ inoculum) diluting the phage fraction 200 times, giving $<0.1 \mathrm{pfu} / \mathrm{mL}$ after 4 transfers. A wash-out of phage after 46 generations could explain the observed increase in the abundance of lineage 1-5. Moreover, in the supernatant of blends 2 and 3, we did not find phages predating on the end-point isolates (Supplemental Figure S3; http://dx.doi.org/10.3168/ jds.2015-9535). The new fraction of strains of lineage 1-5 slowly recovered and gradually increased up to $77 \%$ (blend 2) and 95\% (blend 3) after 152 generations.

In both observed phenomena - initial dominance of lineage 1-5 and initial dominance of lineage 6 - phages seem to affect blend diversity, but after 46 (blends 2 and 3) or 61 (blend 1) generations, the effects of phages weakened and other factors, such as growth rate in milk and the action of cooperative traits ( $\mathrm{prt}^{-}$benefiting from $\mathrm{prt}^{+}$), influence the relative abundance of the lineages. In all 3 phage-challenged blends after 5 to 7 transfers (46 to 61 generations), we observed a trend of increasing abundance of lineage $1-5$ variants. When there were no phages present, this simple culture gravitated toward stable domination of lineage $1-5$. The results obtained in phage-challenged blends suggest that eventually phage-resistant variants survive in these simple communities, as witnessed by their gradual increase in relative abundance, finally leading to a stable situation, as found in blends without phage addition. Our results on phage susceptibility of isolates from the end-point of propagation (152 generations) support this conclusion. Only two strains (from blend 1) out of 60 strains isolated from end-point (20 strains per blend) showed sensitivity to the supernatant obtained from the sample at the end-point (Supplemental Figure S3; http://dx.doi.org/10.3168/jds.2015-9535).

Bacteriophage predation is not the only factor affecting the composition of microbial communities in general and that of complex dairy starters in particular. Many other factors, such as the genetic repertoire of the strains, interactions between strains, competition, and environmental fluctuations, are thought to be important (Smid and Lacroix, 2013), although the relative contribution of each of these factors is difficult to quantify. Yogurt starter cultures represent another example of simple community of 2 interacting species of lactic acid bacteria: Streptococcus thermophilus and Lactobacillus delbrueckii ssp. bulgaricus. The interaction between these 2 yogurt bacteria, known as protocooperation, is crucial for optimal culture functionality and final product characteristics (Sieuwerts et al., 2008; Settachaimongkon et al., 2014). From earlier studies (Hugenholtz et al., 1987; Bachmann et al., 2011), we know that the ratio of protease-positive to proteasenegative variants is crucial for the acidifying activity of the cheese starter culture. Analysis of the protease activity of the isolates used for blend preparation and of the isolates picked up after 152 generations demonstrated that some of the protease-positive strains lost their prt $^{+}$phenotype upon propagation in milk. Our results for blends with phages showed that the niche occupied initially by the lineage 6 strain was partially taken over by $\mathrm{prt}^{-}$variants of lineage $1-5$ strain(s). Hugenholtz and Veldkamp (1985) recognized the presence of protease-negative variants in mixed starter cultures as a separate factor contributing to culture complexity.

The complex cheese starter culture Ur consists of multiple highly related strains representing different co-existing genetic lineages, and the current study demonstrated that strains belonging to the same genetic lineage differ to a large extent in bacteriophage sensitivity. The approach of designing simple blends (i.e., low strain number) was chosen to investigate the influence of phages on culture diversity. The relatively low lineage diversity in these simple blends and exposure to one of the selected phages from the original starter was not enough to sustain maximal diversity at the level of lineages. Further research with defined blends encompassing a larger collection of strains and more diversity resembling the original complex starter culture, combined with predation by multiple types of phages, would allow evaluation and grading of the role of phage predation and other factors in sustaining diversity of the culture upon propagation in milk and its effect on starter functionality (Smid et al., 2014; Smid and Kleerebezem, 2014).

\section{ACKNOWLEDGMENTS}

The project was funded by TI Food and Nutrition (Wageningen, the Netherlands), a public-private partnership on precompetitive research in food and nutrition. The public partners are responsible for the study design, data collection and analysis, decision to publish, and preparation of the manuscript. The private partners contributed to the project through regular discussion.

\section{REFERENCES}

Bachmann, H., D. Molenaar, M. Kleerebezem, and J. E. T. van Hylckama Vlieg. 2011. High local substrate availability stabilizes a cooperative trait. ISME J. 5:929-932.

Daly, C., G. Fitzgerald, and R. Davis. 1996. Biotechnology of lactic acid bacteria with special reference to bacteriophage resistance. Antonie van Leeuwenhoek 70:99-110.

Erkus, O., V. C. de Jager, M. Spus, I. J. van Alen-Boerrigter, I. M. van Rijswijck, L. Hazelwood, P. W. M. Janssen, S. A. F. T. van Hijum, M. Kleerebezem, and E. J. Smid. 2013. Multifactorial diversity sustains microbial community stability. ISME J. 7:2126-2136.

Gómez, P., and A. Buckling. 2011. Bacteria-phage antagonistic coevolution in soil. Science 332:106-109. 
Haegeman, B., J. Hamelin, J. Moriarty, P. Neal, J. Dushoff, and J. S. Weitz. 2013. Robust estimation of microbial diversity in theory and in practice. ISME J. 7:1092-1101.

Holmfeldt, K., M. Middelboe, O. Nybroe, and L. Riemann. 2007. Large variabilities in host strain susceptibility and phage host range govern interactions between lytic marine phages and their Flavobacterium hosts. Appl. Environ. Microbiol. 73:6730-6739.

Hugenholtz, J., R. Splint, W. N. Konings, and H. Veldkamp. 1987. Selection of protease-positive and protease-negative variants of Streptococcus cremoris. Appl. Environ. Microbiol. 53:309-314.

Hugenholtz, J., and H. Veldkamp. 1985. Competition between different strains of Streptococcus cremoris. FEMS Microbiol. Lett. $31: 57-62$.

Huggins, A. R., and W. E. Sandine. 1984. Differentiation of fast and slow milk-coagulating isolates in strains of lactic streptococci. J. Dairy Sci. 67:1674-1679.

Kütahya, O. E., M. J. C. Starrenburg, J. L. W. Rademaker, C. H. W. Klaassen, J. E. T. van Hylckama Vlieg, E. J. Smid, and M. Kleerebezem. 2011. High-resolution amplified fragment length polymorphism typing of Lactococcus lactis strains enables identification of genetic markers for subspecies-related phenotypes. Appl. Environ. Microbiol. 77:5192-5198.

Laan, H., E. J. Smid, P. S. T. Tan, and W. N. Konings. 1989. Enzymes involved in the degradation and utilization of casein in L. lactis. Neth. Milk Dairy J. 43:327-345.

Lillehaug, D. 1997. An improved plaque assay for poor plaque-producing temperate lactococcal bacteriophages. J. Appl. Microbiol. $83: 85-90$

Mahony, J., and D. van Sinderen. 2014. Current taxonomy of phages infecting lactic acid bacteria. Front. Microbiol. 5:7.

Mc Grath, S., G. F. Fitzgerald, and D. van Sinderen. 2007. Bacteriophages in dairy products: pros and cons. Biotechnol. J. 2:450-455.

McKay, L. L., and K. A. Baldwin. 1975. Plasmid distribution and evidence for a proteinase plasmid in Streptococcus lactis C2. Appl. Microbiol. 29:546-548.

Rodriguez-Valera, F., A.-B. Martin-Cuadrado, B. Rodriguez-Brito, L. Pasic, T. F. Thingstad, F. Rohwer, and A. Mira. 2009. Explaining microbial population genomics through phage predation. Nat. Rev. Microbiol. 7:828-836.

Sanders, M. E., and T. R. Klaenhammer. 1983. Characterization of phage-sensitive mutants from a phage-insensitive strain of Streptococcus lactis: Evidence for a plasmid determinant that prevents phage adsorption. Appl. Environ. Microbiol. 46:1125-1133.
Settachaimongkon, S., M. J. R. Nout, E. C. Antunes Fernandes, K. A. Hettinga, J. M. Vervoort, T. C. M. van Hooijdonk, M. H. Zwietering, E. J. Smid, and H. J. F. van Valenberg. 2014. Influence of different proteolytic strains of Streptococcus thermophilus in coculture with Lactobacillus delbrueckii subsp. bulgaricus on the metabolite profile of set-yoghurt. Int. J. Food Microbiol. 177:29-36.

Sieuwerts, S., F. A. de Bok, J. Hugenholtz, and J. E. T. van Hylckama Vlieg. 2008. Unraveling microbial interactions in food fermentations: from classical to genomics approaches. Appl. Environ. Microbiol. 74:4997-5007.

Smid, E. J., O. Erkus, M. Spus, J. C. M. Wolkers-Rooijackers, S. Alexeeva, and M. Kleerebezem. 2014. Functional implications of the microbial community structure of undefined mesophilic starter cultures. Microb. Cell Fact. 13(Suppl. 1):1-9.

Smid, E. J., and M. Kleerebezem. 2014. Production of aroma compounds in lactic fermentations. Annu. Rev. Food Sci. Technol. 5:313-326.

Smid, E. J., and C. Lacroix. 2013. Microbe-microbe interaction in mixed culture food fermentations. Curr. Opin. Biotechnol. $24: 148-154$

Stadhouders, J., and G. J. M. Leenders. 1984. Spontaneously developed mixed-strain cheese starters. Their behaviour towards phages and their use in the Dutch cheese industry. Neth. Milk Dairy J. $38: 157-181$.

Terzaghi, B. E., and W. E. Sandine. 1975. Improved medium for lactic streptococci and their bacteriophages. Appl. Microbiol. 29:807813.

Thingstad, T. F. 2000. Elements of a theory for the mechanisms controlling abundance, diversity, and biogeochemical role of lytic bacterial viruses in aquatic systems. Limnol. Oceanogr. 45:13201328.

Ward, L. J. H., H. A. Heap, and W. J. Kelly. 2004. Characterization of closely related lactococcal starter strains which show differing patterns of bacteriophage sensitivity. J. Appl. Microbiol. 96:144-148.

Winter, C., T. Bouvier, M. G. Weinbauer, and T. F. Thingstad. 2010 Trade-offs between competition and defense specialists among unicellular planktonic organisms: The 'killing the winner' hypothesis revisited. Microbiol. Mol. Biol. Rev. 74:42-57.

Wouters, J. T. M., E. H. E. Ayad, J. Hugenholtz, and G. Smit. 2002. Microbes from raw milk for fermented dairy products. Int. Dairy J. 12:91-109. 